\title{
A PERCEPTIVE SCALE TOOLKIT FOR NAVIGATING ACROSS LENGTH SCALES
}

\author{
Ipsa Jain and Minhaj Sirajuddin \\ Institute for Stem Cell Science and Regenerative Medicine (inStem) \\ Rajiv Gandhi Nagar, Kodigehalli \\ Bengaluru-560065 \\ Karnataka \\ +91-80-6194-8133 \\ minhaj@instem.res.in
}

\begin{abstract}
A naked human eye can perceive objects down to a millimeter length. While lenses and microscopes have overcome this limit, the human mind still lacks perspective when navigating conventional scales (1), especially in the range that are less palpable to naked human eye $(2,3)$. This problem is particularly acute in the context of science communication, where the conventional scale bar units facilitate little comprehension regarding the perception for factorial size differences (3). Here we aim to bridge the gap of scale factors and perspectives using a universal toolkit of objects, which can help comprehend the relative change in length dimensions up to 13 orders of magnitude difference. We further have demonstrated the use of such a universal object toolkit as a length perceptive scale by illustrating and narrating biological phenomena. The meter to picometer 'length perceptive scale' proposed here has the potential to cover majority of length scales present in the biological realm, and is analogous to the time compression methods widely used in explaining cosmos timeline (4). Our toolkit can also be calibrated according to the users need in their scientific communication and illustrations, which will aid the readers' benefit in understanding the length scale perception of illustrated phenomenon.
\end{abstract}

Keywords Science Communication $\cdot$ Scale $\cdot$ Education $\cdot$ Toolkit

\section{Introduction}

The physical limit of a naked human eye can be matched to objects down to a millimeter length. This limit has been overcome by inventions that manipulate light i.e., lenses and microscope, which eventually opened up the microscopic world to human eye. The microscopic images often accompany a gradation factor, a standard way of expressing scale (1). The scale can either be a fixed SI length unit scale bar called a nominal scale, or ratio scale factor, which provides the order of variation; a zoom factor in case of microscopes images.

Biological processes often occurring at the tiniest possible scale can trigger cascade of events, 
which is then amplified and manifested into the visible realm at an organismal level. Although we are able to peer through these tiniest possible objects and integrate them at different scale levels (2), the human mind lacks perception when navigating scales at the range that are less palpable to naked human eye. This becomes particularly problematic in scientific illustrations where most of them are flattened representations of a 3-D shapes and forms. For instance, if one takes an example of reproductive cycle of a fruiting plant and attempts to illustrate the process, the scheme will roughly follow; a blooming adult tree, pollen transfer, fertilization, gamete fusion, embryogenesis, and fruit development (5). In the due course we would have navigated 11 orders of magnitude, roughly from meter $\left(10^{0} \mathrm{~m}\right)$ i.e., adult tree to Angstrom units $\left(10^{-10} \mathrm{~m}\right)$ e.g., protein-protein interactions during pollination. A scale bar in each image will provide an absolute sense about dimension and once below millimeter (1/1000 of a meter), the micrometer (1/1000000 of a meter) and nanometer (1/1000000000 of a meter) in units become less sensible and clouds our perception of length dimensions. A less tedious version is to use zoom factor, however without a reference the ratio scale factor also suffers similar lack of comprehension as nominal scale.

Even at the perceptible scale range (i.e., between kilometer to millimeter) navigating between unknown objects that are roughly orders of magnitude with each other can be hard to discern. For example, picture yourself sitting on an airplane next to a window seat. During takeoff you will observe objects in the ground shrinking and at some point the size differences of objects is lost at different heights. While this example refers to change in distance between the observer and the object viewed, the loss in perception of scale is analogous to the one observed while navigating through images across scales.

To mitigate these proportional issues authors, educators, science communicators and illustrators often use contextual information or a reference scale unit to depict the magnitude of illustrated object. In written articles it is common to apply the equivalence depictions using tall buildings, football fields, city blocks, countries or states etc., This has captivating effect to understand the magnitude of change for e.g., during the 2018 California wildfires, the fire spread rate was reported as roughly a football field every second (6). In the case of pictures, especially related to natural history and paleontological enquiry, commonly used materials such as rulers, coins, pins, pens and hand etc., are used to accurately represent the size of the pictured subject. For scientific illustrations spanning across different log scale dimensions, a ten million times (TMT) perceptive scale was proposed recently (3). The TMT perceptive scale suggests cellular components to be magnified up to the size of daily used objects and use them as a scale factor, which allows the user to narrate the cellular processes up to five log folds of dimensions (from a molecular to a sub-cellular scale). However, the TMT scale falls short in visual narratives that span several log folds, from whole organism (meter, $100 \mathrm{~m}$ ) down to molecular scale (Angstrom, 10 ${ }^{-10} \mathrm{~m}$ ).

There are several resources available that exemplifies the order of magnitude length scale changes; Cosmic view in 40 jumps (7) and its related powers of ten video (8), and other pedagogical tools $(9,10)$. However, it has not been applied as contextual information to depict length scale differences across several orders of magnitude. Here we have assembled a list of universal objects that have defined dimensions and sorted them in a transcending order, which can be applied as a proxy for depicting the length scale navigation and visual narratives spanning from meter $\left(10^{0} \mathrm{~m}\right)$ to picometer $\left(10^{-12} \mathrm{~m}\right)$. 

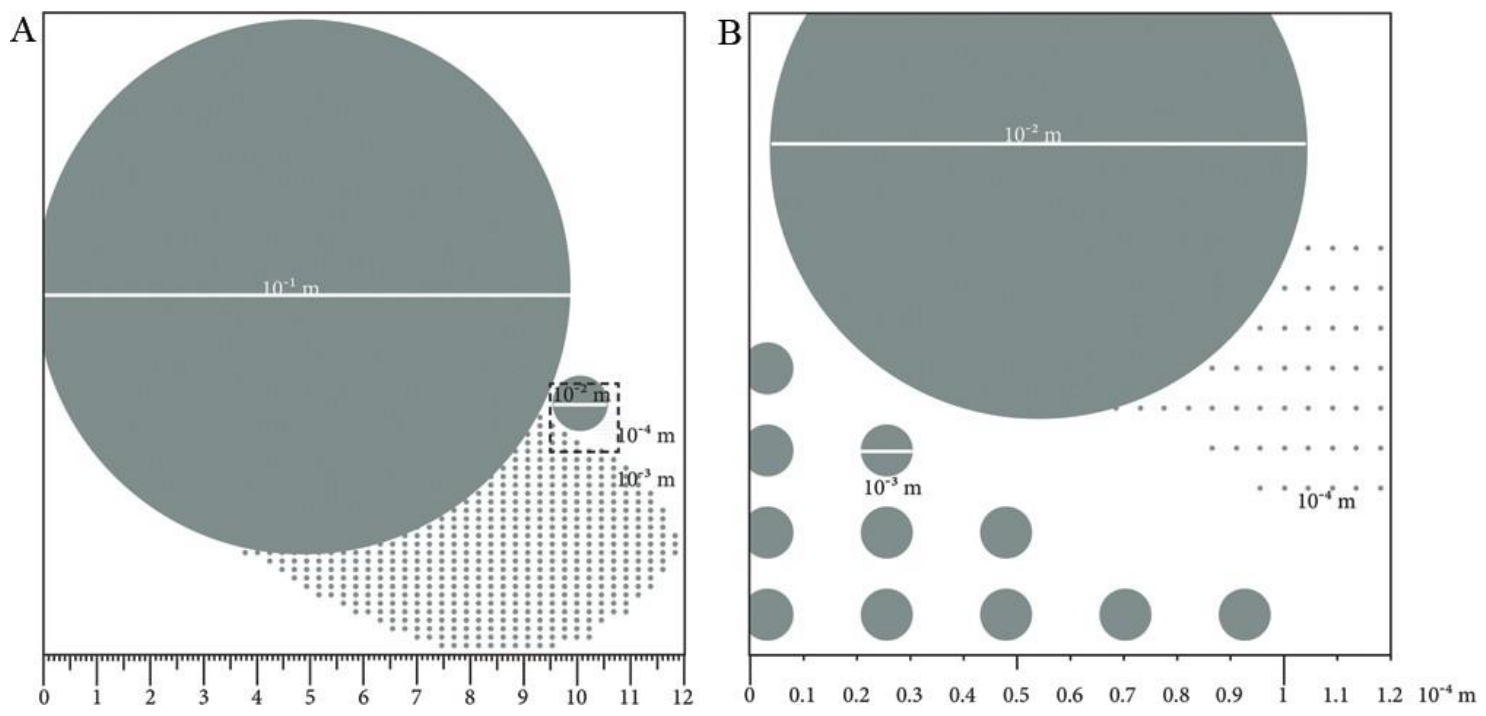

Figure 1: Limitations of resolvable power. (A) Circles of $10^{-1}(10 \mathrm{~cm}), 10^{-2}(1 \mathrm{~cm}), 10^{-3}(1 \mathrm{~mm})$ and $10^{-4}(0.1 \mathrm{~mm})$ meters were placed next to each other, illustrating the 4 orders magnitude changes and limitations in resolving power in a single image. (B) 10 times zoomed view of A.

\section{Power of 10 objects}

One of the major challenges in illustrations of any nature is the inability to depict several log fold objects in a single frame. Artistic license has allowed illustrators to depict very wide range of largescale dimensions in one frame/image/page. This is most exemplified with our solar system illustrations that span all the planetary systems including the Sun. Even if we ignore the distances for illustrative purposes, the sizes of planets are grossly misrepresented. Here we have attempted to place Sun, Saturn, Earth and Moon in a single illustration without compromising their factorial size differences (Supplement Figure 1). The diametrical dimensions of these planetary objects are $1.39 \times 10^{9} \mathrm{~m}$ (Sun), $1.20 \times 10^{8} \mathrm{~m}$ (Saturn with rings), $1.27 \times 10^{7} \mathrm{~m}$ (Earth) and $3.47 \times 10^{6} \mathrm{~m}$ (Moon), which roughly scales down to an order of magnitude in meters. Since these planetary objects are beyond the perceptive scale and realistically not possible to put them in a single picture, we then attempted to place circles with a range of 4 orders of magnitude size changes, $10 \mathrm{~cm}\left(10^{-1}\right.$ $\mathrm{m}), 1 \mathrm{~cm}\left(10^{-2} \mathrm{~m}\right), 1 \mathrm{~mm}\left(10^{-3} \mathrm{~m}\right)$ and $0.1 \mathrm{~mm}\left(10^{-4} \mathrm{~m}\right)$ (Figure $\left.1 \mathrm{~A}\right)$. While the $0.1 \mathrm{~mm}$ circles are barely visible in Figure 1A, the same picture when zoomed in $10 \mathrm{X}$, the $0.1 \mathrm{~mm}$ circle begins resolvable, however the $10 \mathrm{~cm}$ circle, which is 1000 times larger than the $0.1 \mathrm{~mm}$ becomes out of context (Figure 1B). Therefore, it becomes clear that in a single image it is realistically not possible to depict beyond 4 orders of magnitude and becomes the upper limit of human eye resolving power.

In the light of this limitation, we wondered if there is a range of universal objects (as described in the Supplement Figure 1) where one could capitalize the order differences for a new perceptive scale. We then assembled a list of objects with fixed dimensions starting from the largest object in the solar system, the Sun $\left(1.39 \times 10^{9} \mathrm{~m}\right)$ i.e., 1.39 billion meters and decreasing the order dimension down to the tip of a ballpoint pen $\left(10^{-3} \mathrm{~m}\right)$ i.e., $1 / 1000$ of a meter or 1 millimeter (Table 1). The second largest object in diametrical dimension is Jupiter $\left(1.4 \times 10^{8} \mathrm{~m}\right)$ and we also chose Saturn $\left(1.27 \times 10^{8} \mathrm{~m}\right.$, including the ring diameter) in the same order category, as it is the closest to an order 
below and above to Sun and Earth respectively. Another advantage of Saturn is its recognizable rings, which will serve well as icon placed in an illustration. Following the Earth, Moon $\left(3.47 \times 10^{6}\right.$ $\mathrm{m})$ or Ceres $\left(0.97 \times 10^{6} \mathrm{~m}\right)$ and continents represent objects that are in a million-meter range. The list tapers down in dimension to famous Earth structures both manmade and natural; Suez and Panama canals (hundred thousand meters), Mount Everest (ten thousand meters), Diameter of Large Hadron Collider and Golden Gate bridge (thousand meters). Hundred meters and below we have several standard sized fixed object examples; sports and athletic fields (hundreds and tens of meters), bicycle and human (meters) and standard sized sports balls (sub-meters). Finally we end the scale in millimeter range with examples of coins, pen tips and ball bearings. The objects chosen here represents size wise dimension in diameters, they are fixed in size and have less scope of size changes in future (Table 1).

The range from billion meters to millimeter respectively using Sun to pen tip as reference is 13 orders of magnitude in length scale. Which can be used as a proxy to represent meter to picometer dimension, hence we propose a meter to picometer (MPM) length perceptive scale (Table 1 and Figure 2). The proposed MPM scale is a perceptive scale and therefore cannot be used to represent the true scale of illustrated object such as a reference scale. However the MPM scale becomes valuable when navigating orders of magnitude changes in scientific illustration. Similar to a reference scale, the MPM length perceptive scale can provide contextual information and aid the reader in understanding processes spanning several orders of magnitude changes.

\section{Narration using MPM perceptive scale}

Armed with a length perceptive scale we attempted to apply this in explaining biological phenomena, which requires navigation across many orders of scale. Here we have illustrated the life cycle of an apple tree, from an adult tree to seed there are several events occurring at various dimensions. From flower to cross-pollination by bees we have already jumped 4 orders of magnitude (1000X) in detail and becomes the upper limit in depiction as described earlier (Figure 1 and Supplement Figure 1). Therefore, we split the image panels zooming down/out in each as we navigate across the scales with appropriate perceptive reference for each frame (Figure 3 ).

In the early spring, the leafless apple tree shows signs of activity and before the leaves shoot the tree starts to blossom. Even though the flowers are visible from far apart, a buzz of activity is happening at a very close range. Bees, the chief pollinator bring in pollen from different flowers, cross-pollinate and aid in the fertilization. A cascade of events is triggered at a very tiny scale and our visual realm ends at scale of anther (the sack containing pollen). The pollen once in place; on the surface of stigma, the 'female' receptacle extends the tube down to ovary. Here the selection or discrimination of self versus foreign pollen happens at molecular level, where the protein-protein interaction dictates if the pollen tube will extend all the way to the ova, deep inside the stigma, thereby ensuring the fertilization $(5,11)$. Once fertilization is successful the seed forms and the tree starts to make apples.

An important element here is the molecular lock-key feature of receptor interaction at Angstrom scale, which needs to be set right for fertilization and further fruit development. This cascade of activity is almost to the scale equivalent to a footballer kicking the ball into opponents' goalpost (receptor incompatibility) to trigger fan euphoria in the stadium (cell to cell interaction), which is then amplified to whole nation or continent (apple formation). In the event of receptor compatibility 


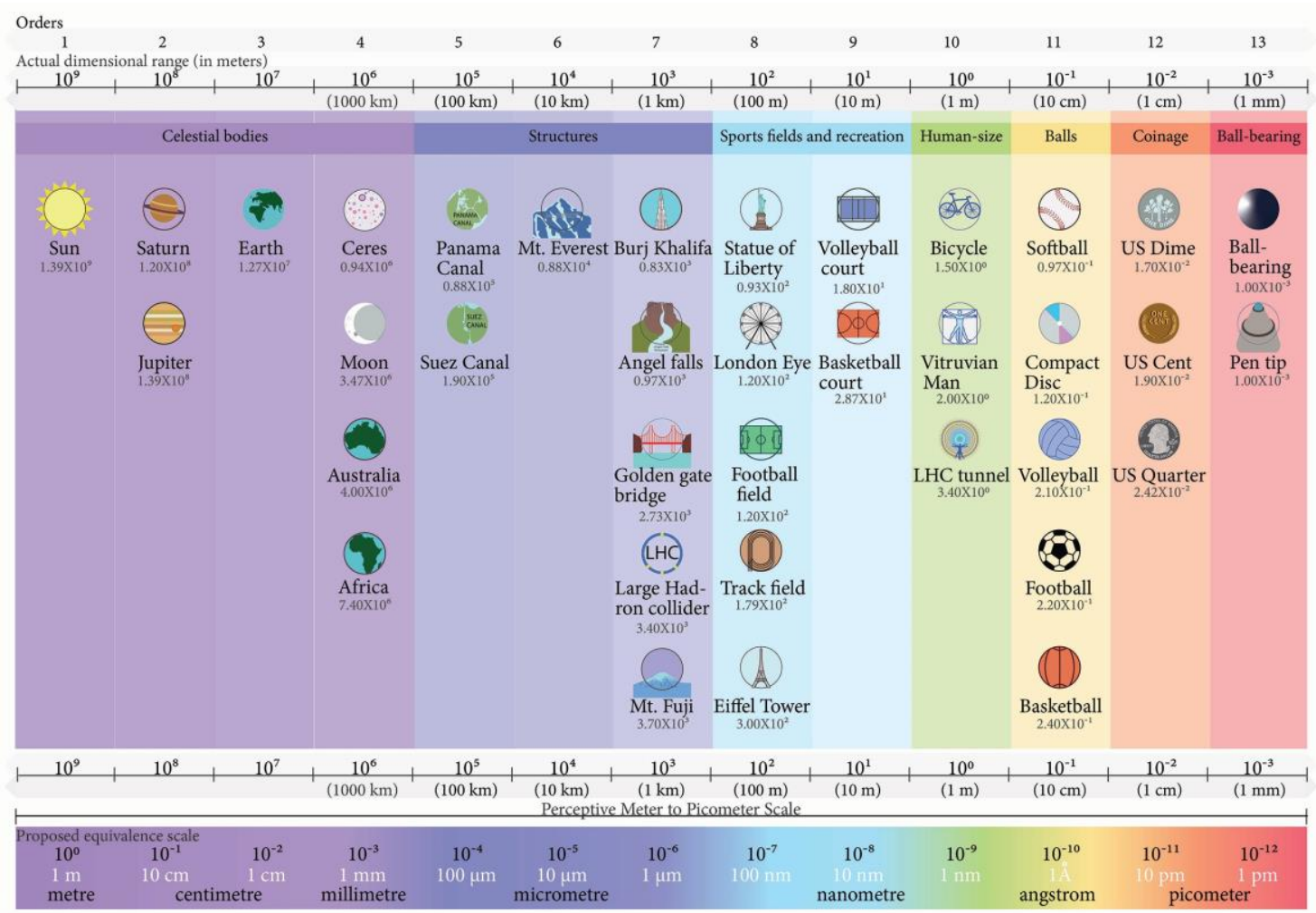

Figure 2: Meter to picometer perspective scale. The universal objects illustrated here extend from the largest in solar system, Sun $\left(10^{9} \mathrm{~m}\right)$ to millimeter objects $\left(10^{-3} \mathrm{~m}\right)$. These 13 orders of objects are divided into celestial bodies, earthly structures, sports and recreation, human-sized, balls, coinage and ball bearings. A relative scale of 13 order of magnitude range (from meter to picometer) is placed beneath the actual dimensional range of objects, which represents the proposed perceptive or equivalence scale. For more detailed list see Table 1.

(self-goal), the activity becomes lull and agonistic. Thus, the accompanying perceptive scale along with narration enhances the comprehension of length scales.

While the apple tree life cycle goes back and forth in length scale with a time component, we used another biological example, which occurs in real time and involves cascade triggered by light to generate currents in neurons of the eye (that communicate with brain for image formation). Here the light is converted in to electrical signal and passed on to the brain. While the information in the form electrical signal is passed few centimeters from eye to brain, the signal conversion occurs at molecular level. As one goes deeper into the eye, passing the lens and vitreous cavity, light hits the retina, a highly stratified layer of eye tissue. The stratification includes non-interfering bipolar cells, ganglion cells, horizontal cells nerve cells, a convoluted layer of rod and cone cells and a mixture of cell and matrix material. The membranes of rod cells have tiny molecular machines, which can respond to light and trigger an avalanche of response by charged elements (ions that are $10^{-10} \mathrm{~m}$ ) to generate electrical signals (12) (Figure 4).

The electrical signal generation is analogous to tripping a penny-sized switch (retinal isomerization by light), to open turbine tunnels (ion channels) for producing electricity in a hydroelectric plant (rod cells of retina), transporting electricity in grid lines (neurons) for a faraway city (brain). Here we have we have added perceptive scale along with absolute scale to a different calibration than that 


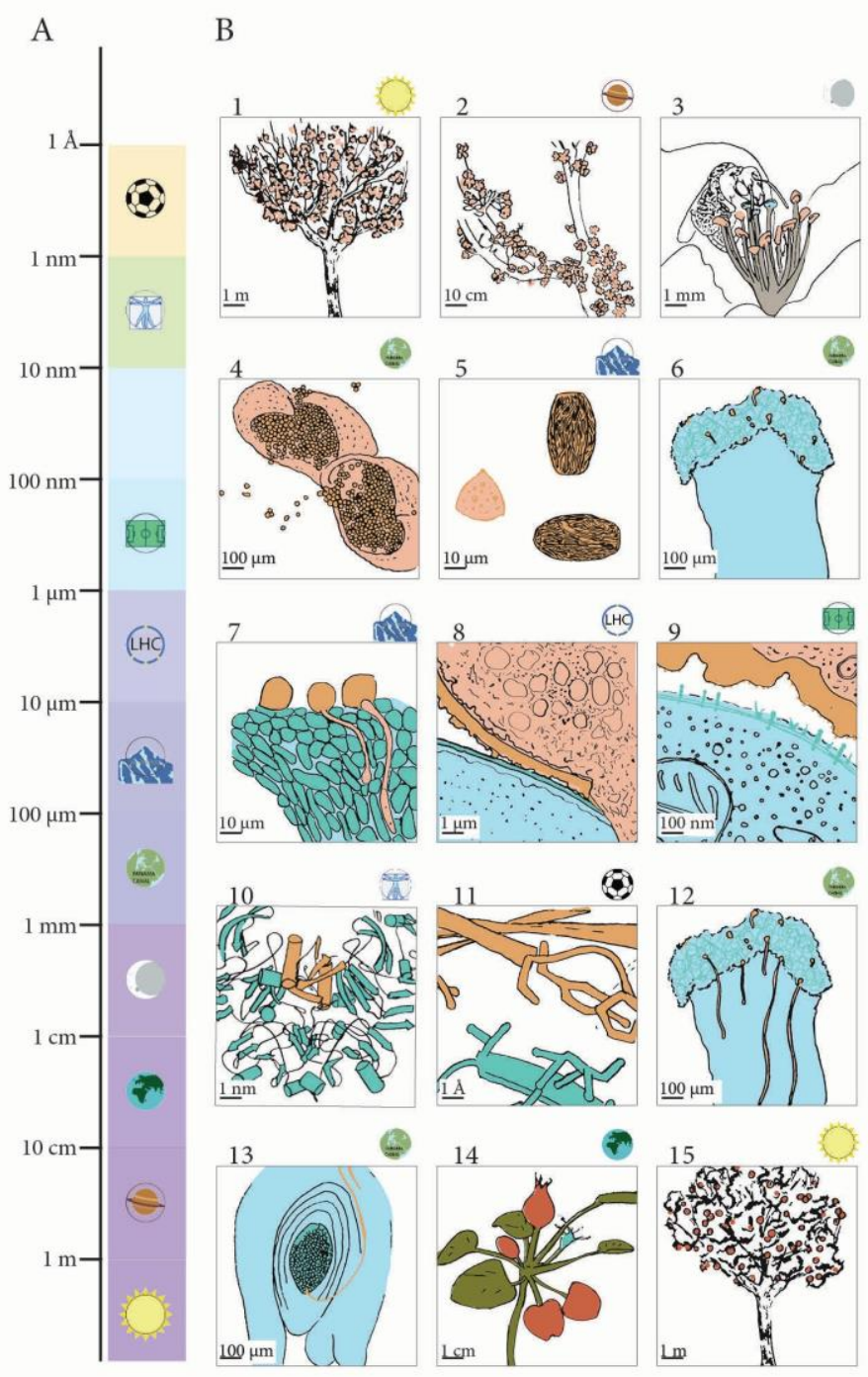

Figure 3: Life cycle of an apple tree. (A) 11 order perceptive scale ranging from meter (Sun) to Angstroms (Football). (B) Illustration of apple fruit formation process using $5 \times 3$ panels, each representing a scale dimension. (1) An apple tree blossom in the spring season. (2) and (3) The flower, hermaphrodite-with both male (orange) and female (cyan) parts. Bees transfer pollens across flowers (cross-pollination). (4) and (5) Closer view of anthers, the pollen sack and individual pollens with rough surfaces with and 'sperm or gamete material' packaged inside them. (6) The bee deposited pollens adhere to stigma of the flower, the female receptacle. (7) Closer look of the pollen tube extending to pass into the stigma. (8) and (9) The interaction between the pollen and the female receptacle determine the fate of the pollen tube. (10) and (11) This is determined at molecular level where the ligand from pollen (orange protein) should not match receptor from female receptacle (cyan protein) (PDB ID: 5GYY), in case of match as illustrated here the pollen is destined for self-destruction. This lack of fidelity is important to ensure cross-pollination and is kept in check by chemical bond interactions at Angstroms scale. (12) and (13) Once the genetic diversity is ensured the pollen tube extends all the way to ovum for fertilization. (14) and (15) Within weeks of post-fertilization the seeds (plant embryo) are packaged inside a fleshy fruit for seed dispersal. 
Perceptive Scale

A

\begin{tabular}{|c|c|c|c|c|c|c|c|c|c|}
\hline $10 \mathrm{~cm}$ & $1 \mathrm{~cm}$ & $1 \mathrm{~mm}$ & $100 \mu \mathrm{m}$ & $10 \mu \mathrm{m}$ & $1 \mu \mathrm{m}$ & $100 \mathrm{~nm}$ & $10 \mathrm{~nm}$ & $1 \mathrm{~nm}$ & $1 \AA$ \\
\hline
\end{tabular}

B
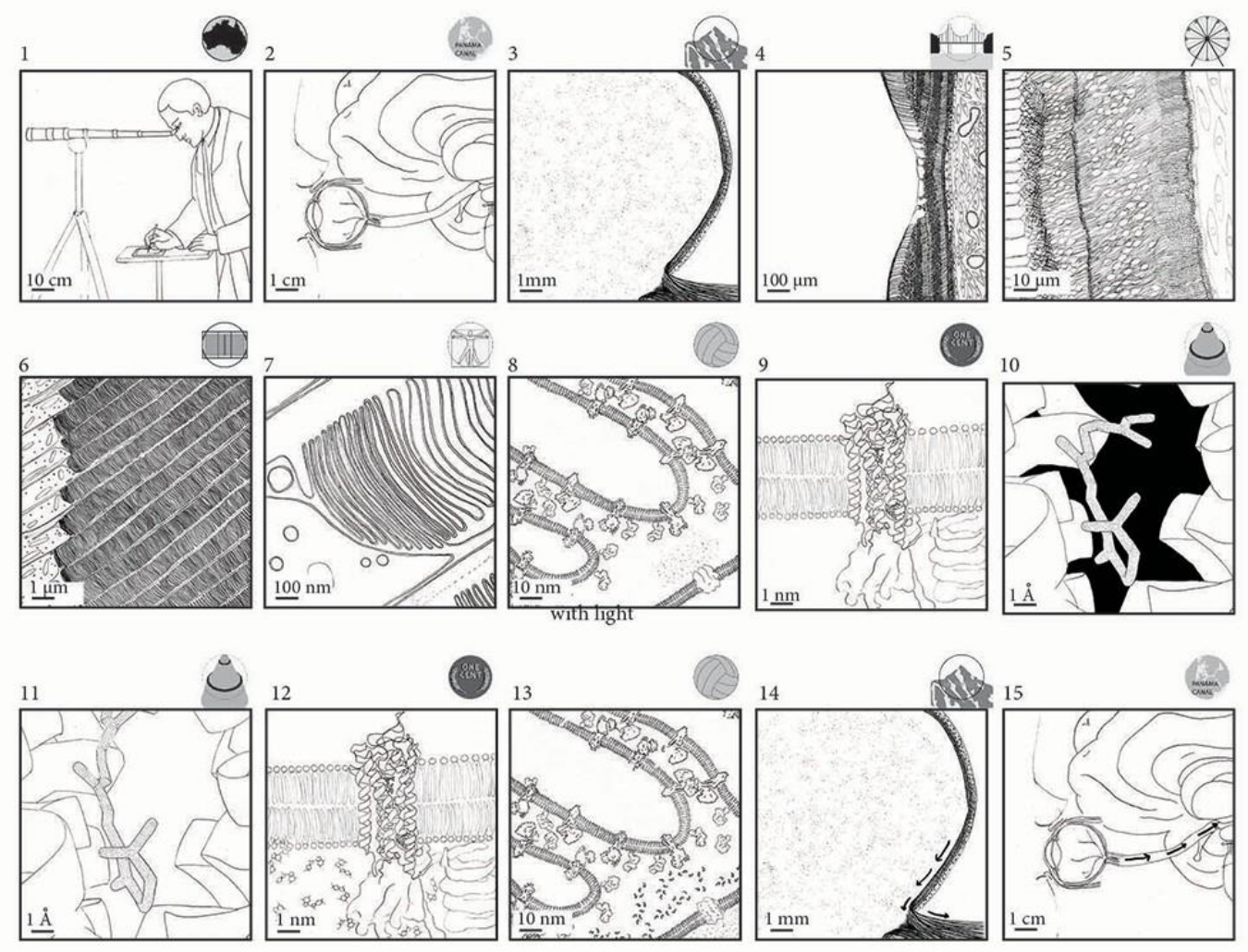

C

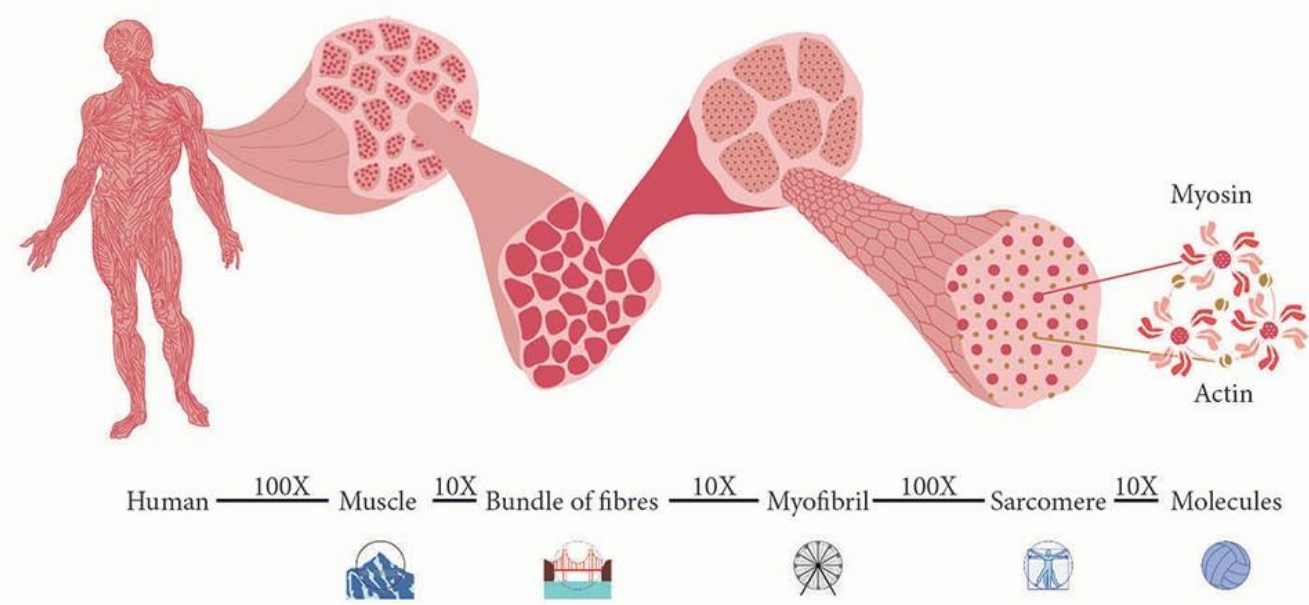

Figure 4: Light to electrical signal processing in eye. (A) 10 order perceptive scale ranging from decimeter (Australian continent) to Angstroms (pen tip). (B) Illustration of how light is converted into electrical signals for brain using $3 \times 5$ panels, each representing a scale dimension. (First row) When a human eye gazes towards illuminated material, the visible light range (blue to red) enters the eye lens, light gets focused on projected into the retina. The stratification of retina tissue is shown in the first row rightmost panel; in the retina there are three layers of cells. The light passes through the ganglion cells, the bipolar cells, the interspersed horizontal cells, finally to the light sensitive layer of rods and cone cells. Next to these cells, is a layer of small melanocytes, followed by connective tissues. The axons of the nerve cells bundle up and become the optic nerve that communicates the signal to the brain. The morphology of rod and cone cell is a stalk like cell, with a bulb at the light facing side and a brush like structure facing away from the light 
Perceptive Scale

of apple story (Figure 3). Thus exemplifying the flexibility of 'power of 10' objects listed for length perceptive scale (Table 1).

With these two examples we show that supplementing illustrations with a length perceptive scale and narration enhances the visual representation by aiding the readers to comprehend the length scale navigation.

\section{Pros and cons}

The proposed length perceptive scale and the list containing power of 10 objects has wide-range of applications and advantages than described above. The reference images or icons can also be placed in the images instead of a scale bar. For this purpose we have also provided a downloadable panel of vector-based icons as listed in Table 1 (Supplement material). Because the reference icons used here are mostly circular and symmetrical in shape, these objects can give clues about dimensionality with respective to diameter and area compared to the nominal scale. Some of the icons presented here have different multiplication factors for a given order of scale, which the users can scale according to the exact multiplication factor for that magnitude.

Although in our example storyboards we have used image panels zooming in with each frame, our perceptive scale will benefit perspective drawings. Scientific illustrations often use perspectivebased drawings where the resolution increases in one direction, exemplifying the continuity in architecture, however distorting the scale e.g., chromatin architecture (14), neuronal cells (15) and muscle fibers (13) (Figure 4C). In such illustrations it is possible to provide the right dimensional cues with the use of proposed perceptive scale. By placing the right reference object near to image subjects at each scale order jump will facilitate dealing with the scale distortion (Figure 4C).

Another major advantage is the gradable nature of perceptive scale, which we have illustrated in the apple and light sensing storyboards. In addition, there exist many pair of objects or gradation pairs, which can be clubbed together, to better illustrate the scale magnitude difference. For example, Sun versus Saturn versus Earth (each 1 order difference), Basketball or Volleyball and its respective court (2 orders), Moon versus LHC versus LHC tunnel (each 3 order differences), humans and Mt. Everest (4 orders), etc., We have illustrated many such pairs (Supplement Figure 2 ) and we encourage users to pair many such according to their needs and requirement.

Figure 4 continued: source, which is cylindrical in rod cells and conical in cone cells. The center of retina, fovea centralis is enriched in cones that detect color in RGB, the other regions have higher density of light detecting rod cells. (Second row) As we zoom into the brush region of the rod cells, we see up to thousands of stacks of intracellular membranous sacs. These sacs have a cavity on the inside and sandwich a thin sliver of cytoplasm between them, which house most of the molecular machinery involved in the light transduction process. The sacs originate as finger like projections, which are later internalized in the cell. The membranes of the disc in rod cells house the rhodopsin molecule that is extremely light sensitive. In the vicinity of rhodopsin exists few other enzymes and ion channels, which are in place to relay the signal. Zoomed in view of a single rhodopsin and adjacent enzymes, the ligand (11-cis-retinal) is at the heart of the rhodopsin and is inactive in dark state. (Third row) When light hits the eye, the retinal is isomerized to trans-retinal. The light induced isomerization of retinal leads to changes in rhodopsin, which is relayed to enzymes nearby to release new molecules in the cell, which then opens the ion channels to create a gradient of current across membrane. The current is then relayed to the brain for image formation. PDB IDs used 1U19 (Rhodopsin with retinal), 6CMO (Rhodopsin and GPCR), 4ZKF (Phosphodiesterase), 5H3O (cyclin-nucleotide gated ion channel). (C) Perspective based illustration of skeletal muscle organization. The image shows cross sections of indicated structures from muscle (millimeters), bundles of fibers (sub-millimeters), myofibrils (micrometers), sarcomere (sub-micron) and acto-myosin molecules (nanometers). Roughly 6 orders of length scale differences between muscle and molecules, which we have illustrated with icons calibrated to the perceptive scale described in $4 \mathrm{~A}$. 
Perceptive Scale

Regardless of many advantages and applications as with any method, ours also suffers few shortcomings. The first and foremost is the relativity of the scale; although we have named meter to picometer length perceptive scale, the scales we propose here are approximate and can only be used for relativity, not one which gives an accurate sense of measurement. The proposed scale also covers vast spectrum of scales from Sun to tip of pen, where a quantitative estimation may not be obvious in the beginning. However with proper narrational supplement as described in this article, the appreciation of scale difference between a tree and a molecule inside one of its cells becomes apparent. Another disadvantage is that the scale is not useful when one is travelling through smaller spectrum of scale, especially at the larger reference subjects. Therefore, for shorter spectrum of magnitude difference we suggest use of the gradation pairs proposed here (Supplement Figure 2).

\section{Conclusion}

Depicting various length scale objects in illustration is challenging, while this is a well-taught concept in graphic design and art schools, we could not find a good example to exemplify this shortcoming. Here we have demonstrated that it is not possible to depict beyond 4 orders of magnitude changes in a single regular-sized image (desktop screens to A3 prints) (Figure 1). Scientists therefore have cleverly overcome by zooming to show deeper details, a great example is the Cosmic View in 40 jumps (7) and the adapted popular video 'powers of 10' (8). As in the 'powers of 10' video, which illustrates the stunning depiction of details divulging as one zooms in, however at some point the viewer still lose the perception of how deeper one has traversed since the start. Therefore, in this article we have put forward a resource for depicting length scales using a list of universal objects as a length perceptive scale; which is unparalleled to other approaches currently available. Comparably, a time compression method is widely used in narrating the cosmic timeline (4), e.g. history of Earth in 24-hours and the cosmic calendar. We envision that our length perceptive scale can be applied across several orders of magnitude and is useful if one needs to navigate from organism (meter) to molecules (Angstroms). Depicting and narrating the output of nanoscale actions is amplified several orders of magnitude in an organism using universal objects will be hugely beneficial as educational tools.

With ever increasing need and enthusiasm for science communication to lay audience, our length perceptive scale will be invaluable during presentations, narrations and illustrations. The challenge is acute when scientists are required to explain their technology used in unraveling the inner workings of cells at the tiniest possible scale (2). For example, biologists often use microscopes with varying degree of zoom amplification. To simply put in perspective, using our length perceptive scale one can narrate microscopy experiments analogous to satellite imagery. The zooming power of microscopes can be equated to the satellites image capturing ability of Earth objects. A magnifying glass is equivalent of taking a picture of the whole Australian landmass from the satellite. A simple compound microscope can zoom in from millimeter to microns, which is equivalent of zooming to Sydney Opera house. A super-resolution microscope can resolve submicron details, equivalent of satellite zooming to human subject near the Opera house. An electron microscope, which can capture sub-nanometer details, is equivalent of capturing an image of a coin in the human subjects' hand. Such types of narrations are only possible with a length perspective scale described here.

Finally, our approach also sets up a new paradigm where a similar approach for how fast biological processes take place (16), a time perceptive scale and how strong forces are exerted (17), a binding perceptive scale can be developed in future. Together with the applications demonstrated here, we 
Perceptive Scale

believe that our resource will be a great boon for scientists, sci-artists and illustrators who are keen in science communication.

\section{References}

1. Stevens, S. S. On the Theory of Scales of Measurement. Science 103, 677-680 (1946).

2. Goodsell, D. S., Franzen, M. A. Herman, T. From Atoms to Cells: Using Mesoscale Landscapes to Construct Visual Narratives. J. Mol. Biol. 430, 3954-3968 (2018).

3. Zoppè, M. Towards a perceptive understanding of size in cellular biology. Nat. Methods 14, 662-665 (2017).

4. Carl Sagan. The Dragons of Eden. (Penguin Random House LLC., 1977).

5. Ramírez, F. Davenport, T. L. Apple pollination: A review. Sci. Hortic. 162, 188-203 (2013).

6. Judson Jones, C. One of the California wildfires grew so fast it burned the equivalent of a football field every second. CNN (2018).

7. Boeke, K. Cosmic View: The Universe in 40 Jumps - by Kees Boeke. (1957).

8. Charles Eames and Ray Eames. Artworkfor film 'Powers of Ten.' Chicago aerial view, including park and marina. 10p2s. (1977).

9.Genetic Science Learning Center - Utah. Cell Size and Scale. Cell Size and Scale (2016). Available at: https://learn.genetics.utah.edu/content/cells/scale/.

10. Nikon Universcale. Nikon | Universcale. From the nanoworld to the uni-verse - The worlds we measure using our infinite yardstick. (2019). Available at: https://www.nikon.com/about/sp/universcale/scale.htm.

11. Tantikanjana, T. Nasrallah, J. B. Ligand-Mediated cis-Inhibition of Receptor Signaling in the Self-Incompatibility Response of the Brassicaceae. Plant Physiol. 169, 1141-1154, (2015).

12. Kang, Y. et al. Cryo-EM structure of human rhodopsin bound to an inhibitory G protein. Nature 558, 553-558 (2018).

13. Cooper, G. M. Actin, Myosin, and Cell Movement. Cell Mol. Approach 2nd Ed. (2000).

14. Valencia, A. M. Kadoch, C. Chromatin regulatory mechanisms and therapeutic opportunities in cancer. Nat. Cell Biol. 21, 152-161, (2019).

15. Magiera, M. M. et al. Excessive tubulin polyglutamylation causes neurodegeneration and perturbs neuronal transport. EMBO J. 37, (2018).

16. Anderson, J. R. Spanning seven orders of magnitude: a challenge for cognitive modeling. Cogn. Sci. 26, 85-112, (2002).

17. Wikipedia Page. Orders of magnitude (force). Wikipedia (2018).

\section{Supplementary Material}

thttps://drive.google.com/open?id=1ct-VdDiL5ybazOxf0w8Pp-5WdNOPiL6y 


\section{Conflict of Interest Statement}

The authors declare no conflict of interest.

\section{Acknowledgements}

The authors wish to thank the members of the Sirajuddin lab and Sunil Laxman and Carsten Janke for comments on manuscript. This work was funded by inStem core grants, CEFIPRA (5703-1), Wellcome Trust-DBT India Alliance Intermediate Fellow (IA/I/14/2/501533) and EMBO Young Investigator award to MS.

\section{Author Contributions}

IJ and MS conceived the project and wrote the manuscript. 
Table 1: MPM (Meter to Picometer) perceptive scale

\begin{tabular}{|c|c|c|}
\hline Proposed objects & Actual dimension in meters & Scale factor (order) \\
\hline Sun & $1.39 \times 10^{9}$ meters & $9(1)$ \\
\hline Saturn with rings & $1.20 \times 10^{8}$ meters & $8(2)$ \\
\hline Jupiter & $1.39 \times 10^{8}$ meters & \\
\hline Earth & $1.27 \times 10^{7}$ meters & $7(3)$ \\
\hline Ceres (asteroid or dwarf planet) & $0.94 \times 10^{6}\left(1 \times 10^{6}\right.$ meters $)$ & $6(4)$ \\
\hline Moon & $3.47 \times 10^{6}$ meters & \\
\hline Australia (4000 km) & $4.00 \times 10^{6}$ meters & \\
\hline Africa $(7400 \mathrm{~km})$ & $7.4 \times 10^{6}$ meters & \\
\hline Panama Canal (88 km) & $0.88 \times 10^{5}$ meters & $5(5)$ \\
\hline Suez Canal (190 km) & $1.9 \times 10^{5}$ meters & \\
\hline Mt. Everest (8800 meters) & $0.88 \times 10^{4}$ meters & $4(6)$ \\
\hline Mt. Fuji (3700 meters) & $3.70 \times 10^{3}$ meters & $3(7)$ \\
\hline Diameter of Large Hadron collider circle & $3.40 \times 10^{3}$ meters & \\
\hline Golden Gate Bridge & $2.73 \times 10^{3}$ meters & \\
\hline Angel Falls, Venezuela & $0.97 \times 10^{3}$ meters & \\
\hline Burj Khalifa & $0.83 \times 10^{3}$ meters & \\
\hline Statue of Liberty (93 meters) & $0.93 \times 10^{2}$ meters & $2(8)$ \\
\hline London eye (120 meters) & $1.20 \times 10^{2}$ meters & \\
\hline Football field (Soccer field) & $1.20 \times 10^{2}$ meters & \\
\hline Track and field (outdoor) & $1.79 \times 10^{2}$ meters & \\
\hline Eiffel Tower & $3.00 \times 10^{2}$ meters & \\
\hline Volleyball Court (18 meter) & $1.80 \times 10^{1}$ meter & $1(9)$ \\
\hline Basketball court (28.7 meter) & $2.87 X 10^{1}$ meter & \\
\hline Bicycle (1.5 meter) & $1.50 \times 10^{0}$ meter & $0(10)$ \\
\hline Life size Vitruvian Man (Da Vinci) (2.0 meter) & $2.00 \times 10^{0}$ meter & \\
\hline
\end{tabular}


Diameter of LHC tunnel cross section (3.4 meter)

$3.40 \times 10^{0}$ meter

\begin{tabular}{lll}
\hline Softball $(97 \mathrm{~mm})$ & $9.70 \times 10^{-2}$ meter & $-1(11)$ \\
Compact Disc $(120 \mathrm{~mm})$ & $1.20 \times 10^{-1}$ meter \\
Volleyball $(210 \mathrm{~mm})$ & $2.10 \times 10^{-1}$ meter \\
Football (Soccer) $(220 \mathrm{~mm})$ & $2.20 \times 10^{-1}$ meter \\
Basket ball $(240 \mathrm{~mm})$ & $2.40 \times 10^{-1}$ meter & $-2(12)$ \\
\hline US Dime $(17 \mathrm{~mm})$ & $1.70 \times 10^{-2}$ meter \\
US Penny $(19 \mathrm{~mm})$ & $1.90 \times 10^{-2}$ meter \\
US quarter dollar $(24 \mathrm{~mm})$ & $2.42 \times 10^{-2}$ meter \\
\hline Ball Bearing $(1 \mathrm{~mm})$ & $1.00 \times 10^{-3}$ meter \\
Ball-point pen tip $(1 \mathrm{~mm}$ tip $)$ & $1.00 \times 10^{-3}$ meter \\
\hline
\end{tabular}




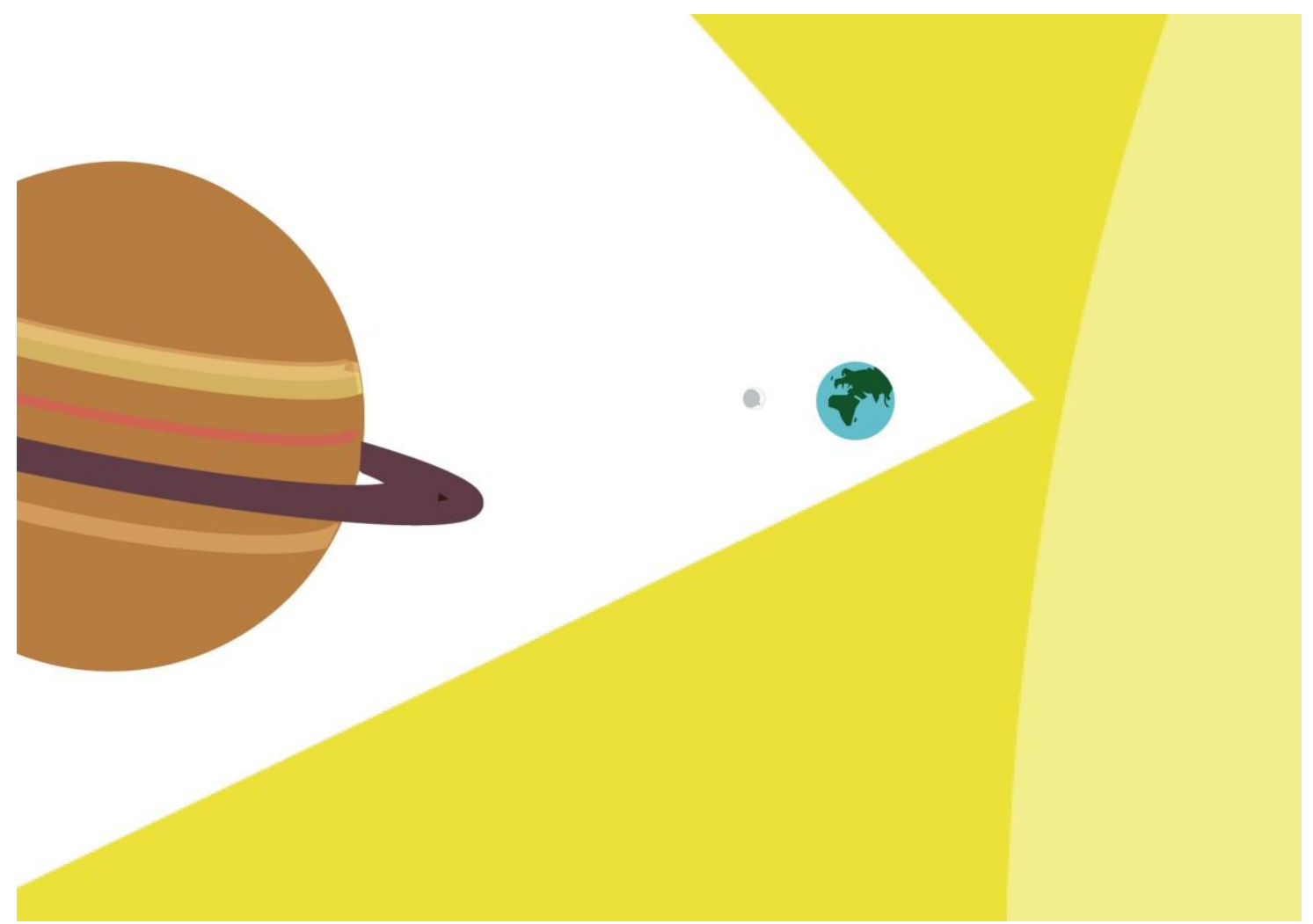

Supplement Figure 1: Ratiometric illustration of Sun, Saturn, Earth and Moon in a single page.

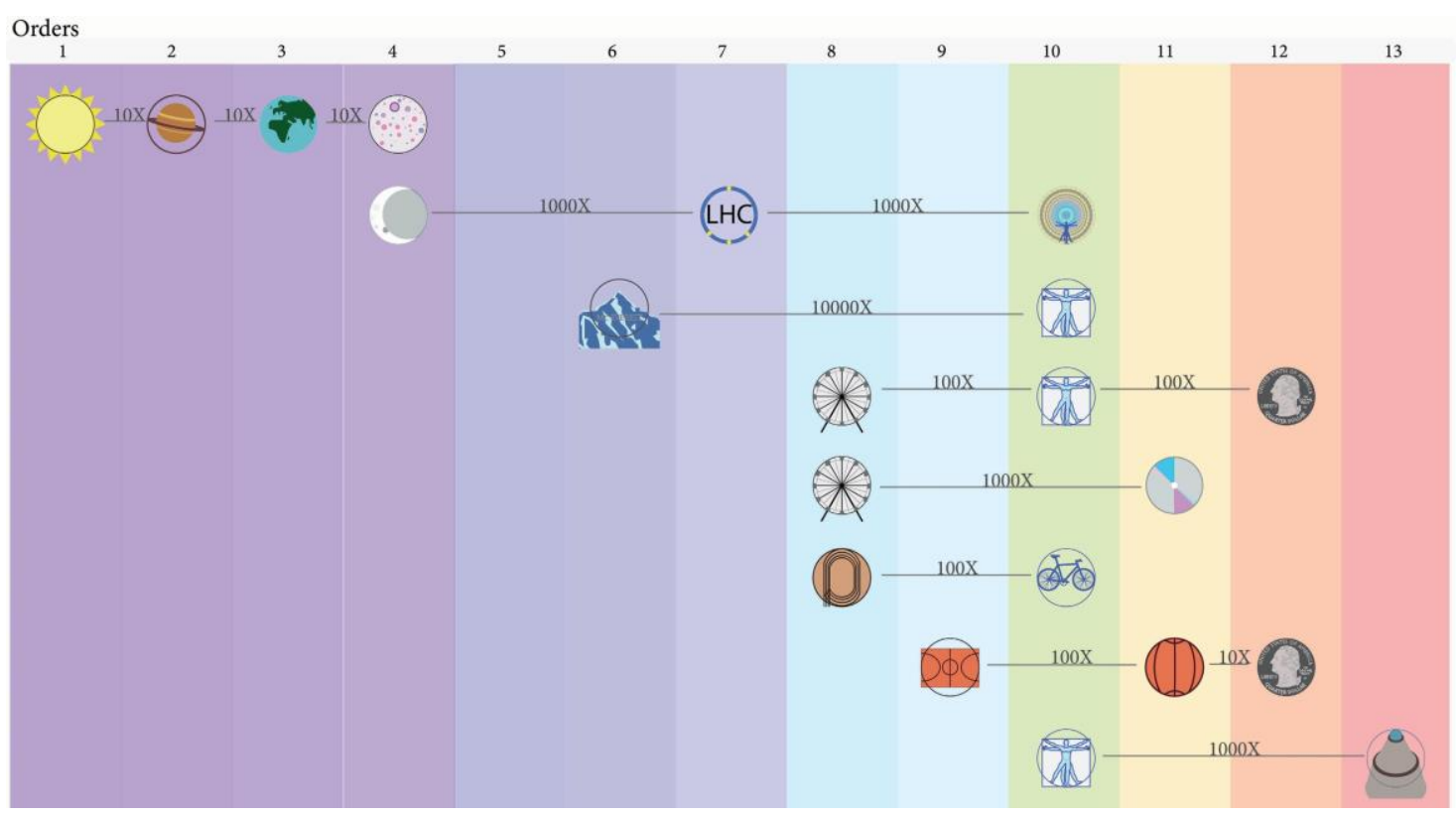

Supplement Figure 2 Equivalence and gradation pair objects with 10, 100, 1000, 10000 times object examples as illustrated. 